\title{
BMJ Open Impacts of type 2 diabetes mellitus and hypertension on the incidence of cardiovascular diseases and stroke in China real-world setting: a retrospective cohort study
}

Yan Liu, ${ }^{1}$ Jie Li, ${ }^{1}$ Ying Dou, ${ }^{2}$ Hongshan Ma (1) ${ }^{3}$

To cite: Liu Y, Li J, Dou Y, et al. Impacts of type 2 diabetes mellitus and hypertension on the incidence of cardiovascular diseases and stroke in China realworld setting: a retrospective cohort study. BMJ Open 2021;11:e053698. doi:10.1136/ bmjopen-2021-053698

- Prepublication history for this paper is available online. To view these files, please visit the journal online (http://dx.doi. org/10.1136/bmjopen-2021053698).

Received 24 June 2021 Accepted 08 November 2021

Check for updates

(C) Author(s) (or their employer(s)) 2021. Re-use permitted under CC BY. Published by BMJ.

${ }^{1}$ Department of Endocrinology, The Third People's Hospital of Datong, Datong, China

${ }^{2}$ Department of Medicine, Ashermed Pharmaceutical Technology Co Ltd, Shanghai, China

${ }^{3}$ Department of Cardiology, The Third People's Hospital of Datong, Datong, China

Correspondence to Hongshan Ma; mahongshannew@163.com

\section{ABSTRACT}

Objective The prevalence of type 2 diabetes mellitus (T2DM) and hypertension (HTN) has notably increased in recent years. However, there is little evidence from largescale studies assessing the joint effect of T2DM and HTN on the risk of cardiovascular events in China. This study was performed to investigate the association of T2DM and HTN with the incidence of combined vascular events (VEs) and stroke in China.

Design A retrospective cohort study.

Setting Data were collected from the SuValue database which includes the electronic medical records of $>90$ million patients from 161 hospitals across 18 provinces in China.

Participants Patients aged $\geq 18$ with a diagnosis of T2DM and/or HTN were included. Non-T2DM and non-HTN patients were included in this study as controls.

Outcomes Incidence of combined VEs and stroke during the study.

Results In the current study, 8012 patients with T2DM, 9653 patients with HTN, 3592 patients with both T2DM and HTN and 10561 patients without T2DM or HTN were included. T2DM was significantly associated with combined VE and stroke risk (HR 1.332, 95\% $\mathrm{Cl} 1.134$ to 1.565 and $\mathrm{HR} 1.584,95 \% \mathrm{Cl} 1.246$ to 2.014 , respectively). HTN was significantly associated with combined VE and stroke risk (HR 3.244, 95\% Cl 2.946 to 3.572 and HR $4.543,95 \%$ Cl 3.918 to 5.268, respectively). T2DM combined with HTN was significantly associated with combined VE and stroke risk (HR 3.002, 95\% Cl 2.577 to 3.497 and HR $4.151,95 \% \mathrm{Cl} 3.346$ to 5.149 , respectively). HTN was associated with a higher combined VE and stroke risk than T2DM (HR 2.435, 95\% Cl 2.113 to 2.805 and $\mathrm{HR}$ $2.868,95 \% \mathrm{Cl} 2.341$ to 3.513 , respectively).

Conclusion T2DM and HTN were strongly associated with combined VE and stroke risk; however, the HTN-only group had a higher combined VE and stroke risk than the T2DMonly group.

\section{INTRODUCTION}

The prevalence of type 2 diabetes mellitus (T2DM) increased to $10.4 \%$ in 2013 and $11.2 \%$ in 2015 from $0.67 \%$ in 1980 in China. ${ }^{12}$ Hypertension (HTN) is found in
Strengths and limitations of this study

This study was designed to analyse the electronic medical records of patients in a real-world setting.

- Cardiovascular risk factors were collected in this study.

- Body Mass Index and lifestyle factors, such as smoking and alcohol consumption, were not recorded in the electronic medical records of patients.

- Mortality data were not accessible through the electronic medical records of patients.

over two-thirds of patients with diabetes. ${ }^{3} 4$ The development of HTN coincides with the development of hyperglycaemia. ${ }^{3}$ Insulin resistance and hyperinsulinaemia might promote atherogenesis, thereby affecting blood pressure homeostasis. ${ }^{56}$

Diabetes has been reported to be a strong risk factor for cardiovascular disease, all-cause mortality, coronary heart disease, ischaemic heart disease and stroke in many studies. ${ }^{7-11}$ According to the Framingham Heart Study, adults with diabetes had an absolute twofold increased risk of cardiovascular disease compared with subjects without diabetes. ${ }^{12}$ The total cardiovascular disease burden in diabetes patients has increased throughout the past four decades. ${ }^{3}$ A cross-sectional study showed that HTN was common in patients with newly diagnosed diabetes, and HTN patients had a higher prevalence of cardiovascular events than normotensive subjects before the diagnosis of diabetes. ${ }^{13}$ HTN has also been reported to be one of the strongest risk factors for cardiovascular disease, including coronary disease, vascular heart disease and cerebral stroke. ${ }^{14-20}$ Adults with coexistent diabetes and HTN dramatically increased the risk of cardiovascular disease by two to four times compared with adults 
Table 1 Baseline characteristics of the included patients

\begin{tabular}{|c|c|c|c|c|c|}
\hline & HTN-only & T2DM-only & T2DM and HTN & $\begin{array}{l}\text { Non-T2DM and non- } \\
\text { HTN }\end{array}$ & $P$ value \\
\hline Total & 9653 & 8012 & 3592 & 10561 & \\
\hline \multicolumn{6}{|l|}{ Sex } \\
\hline Male & $4419(45.8 \%)$ & $4006(50.0 \%)$ & $1634(45.5 \%)$ & $4889(46.3 \%)$ & - \\
\hline Female & $5234(54.2 \%)$ & 4006 (50.0\%) & 1958 (54.5\%) & $5672(53.7 \%)$ & - \\
\hline \multicolumn{6}{|l|}{ Age } \\
\hline $18-29(\mathrm{n}, \%)$ & 79 (0.8\%) & 204 (2.5\%) & 31 (0.9\%) & 1372 (13.0\%) & - \\
\hline $30-39(n, \%)$ & $602(6.2 \%)$ & $831(10.4 \%)$ & $139(3.9 \%)$ & $2439(23.1 \%)$ & - \\
\hline $40-49(\mathrm{n}, \%)$ & $1856(19.2 \%)$ & $2323(29.0 \%)$ & $553(15.4 \%)$ & $3227(30.6 \%)$ & - \\
\hline $50-59(n, \%)$ & $1856(20.7 \%)$ & $2297(28.7 \%)$ & $927(25.8 \%)$ & $1954(18.5 \%)$ & - \\
\hline $60-69(\mathrm{n}, \%)$ & $2552(26.4 \%)$ & $1704(21.3 \%)$ & $1194(33.2 \%)$ & $1179(11.2 \%)$ & - \\
\hline $70-79(\mathrm{n}, \%)$ & $1715(17.8 \%)$ & $556(6.9 \%)$ & $573(16.0 \%)$ & $336(3.2 \%)$ & - \\
\hline$\geq 80(n, \%)$ & $852(8.8 \%)$ & $97(1.2 \%)$ & $175(4.9 \%)$ & $54(0.5 \%)$ & - \\
\hline Triglyceride (mmol/L) & $1.86(1.54)$ & $2.35(2.55)$ & $2.29(1.85)$ & $1.71(1.65)$ & $<0.0001$ \\
\hline Total cholesterol (mmol/L) & $5.31(1.19)$ & $5.32(1.41)$ & $5.29(1.28)$ & $5.28(1.15)$ & 0.3506 \\
\hline $\mathrm{HDL}-\mathrm{C}(\mathrm{mmol} / \mathrm{L})$ & $1.41(0.41)$ & $1.25(0.45)$ & $1.26(0.39)$ & $1.40(0.39)$ & $<0.0001$ \\
\hline LDL-C (mmol/L) & $3.17(1.00)$ & $3.07(1.03)$ & $3.02(0.98)$ & $3.03(0.90)$ & $<0.0001$ \\
\hline Serum creatinine $(\mu \mathrm{mol} / \mathrm{L})$ & $81.11(39.35)$ & $70.57(23.94)$ & $84.26(59.38)$ & $76.78(20.42)$ & $<0.0001$ \\
\hline HbA1c (\%) & $5.58(1.38)$ & $9.41(3.11)$ & $8.41(2.63)$ & $5.73(1.31)$ & $<0.0001$ \\
\hline Fasting blood-glucose (mmol/L) & $5.76(1.28)$ & $11.15(5.28)$ & $9.88(5.14)$ & $5.58(1.13)$ & $<0.0001$ \\
\hline Serum insulin (pmol/litre) & $95.63(60.05)$ & $117.64(288.36)$ & $78.43(57.89)$ & $9.98(3.21)$ & 0.6502 \\
\hline Proteinuria (positive) & $310(3.2 \%)$ & $145(1.8 \%)$ & $126(3.5 \%)$ & $143(1.4 \%)$ & $<0.0001$ \\
\hline \multicolumn{6}{|l|}{ Diabetes complications } \\
\hline Diabetic nephropathy & - & $450(5.6 \%)$ & $446(12.4 \%)$ & - & - \\
\hline Diabetic retinopathy & - & $313(3.9 \%)$ & $159(4.4 \%)$ & - & - \\
\hline Diabetic neuropathy & - & $462(5.8 \%)$ & $225(6.3 \%)$ & - & - \\
\hline $\begin{array}{l}\text { Diabetic lower limb vascular } \\
\text { disease }\end{array}$ & - & $7(0.09 \%)$ & $15(0.42 \%)$ & - & - \\
\hline Diabetic foot & - & $80(1.0 \%)$ & $61(1.7 \%)$ & - & - \\
\hline
\end{tabular}

For continuous variables, data were presented as mean (SD) and $p$ values for four groups were calculated using analysis of variance test. For categorical variables, data were presented as number (frequency) and $p$ values for four groups were calculated using $\chi^{2}$ test.

HDL-C, high-density lipoprotein cholesterol; HTN, hypertension; LDL-C, low-density lipoprotein cholesterol; T2DM, type 2 diabetes mellitus.

without HTN or diabetes. ${ }^{3}$ Thus, diabetes and HTN are thought to be poor companions, and blood pressure control is critical in diabetes patients with HTN. In addition, a study demonstrated that HTN had a stronger association with atherosclerotic cardiovascular disease than diabetes. $^{21}$

The effects of HTN and diabetes on the risk of cardiovascular disease have been investigated in American, Finnish, Japanese and Iranian populations. ${ }^{21-24}$ However, there is little evidence from large-scale studies assessing the joint effect of HTN and diabetes on the risk of cardiovascular events in China. The purpose of the study was to evaluate the impact of HTN and T2DM on the risk of cardiovascular disease and stroke in Chinese adults using the SuValue database.

\section{METHODS}

\section{Study design}

The SuValue database is a big-data hospital information system (HIS) database that includes data on $>90$ million patients from 161 hospitals across 18 provinces in China. ${ }^{25}$ This was a retrospective cohort study designed to evaluate the risk of cardiovascular diseases and stroke in patients with T2DM and/or HTN from 2004 to 2015 in a real-world Chinese setting. Patients were included if they met the following criteria: (1) aged $\geq 18$; (2) newly diagnosed T2DM and/or HTN; (3) had baseline examination records before or within 3 months at the first diagnosis (details of baseline examination were described in the Baseline Parameters section) and (4) electronic medical records (EMRs) could be found 1 year later after 


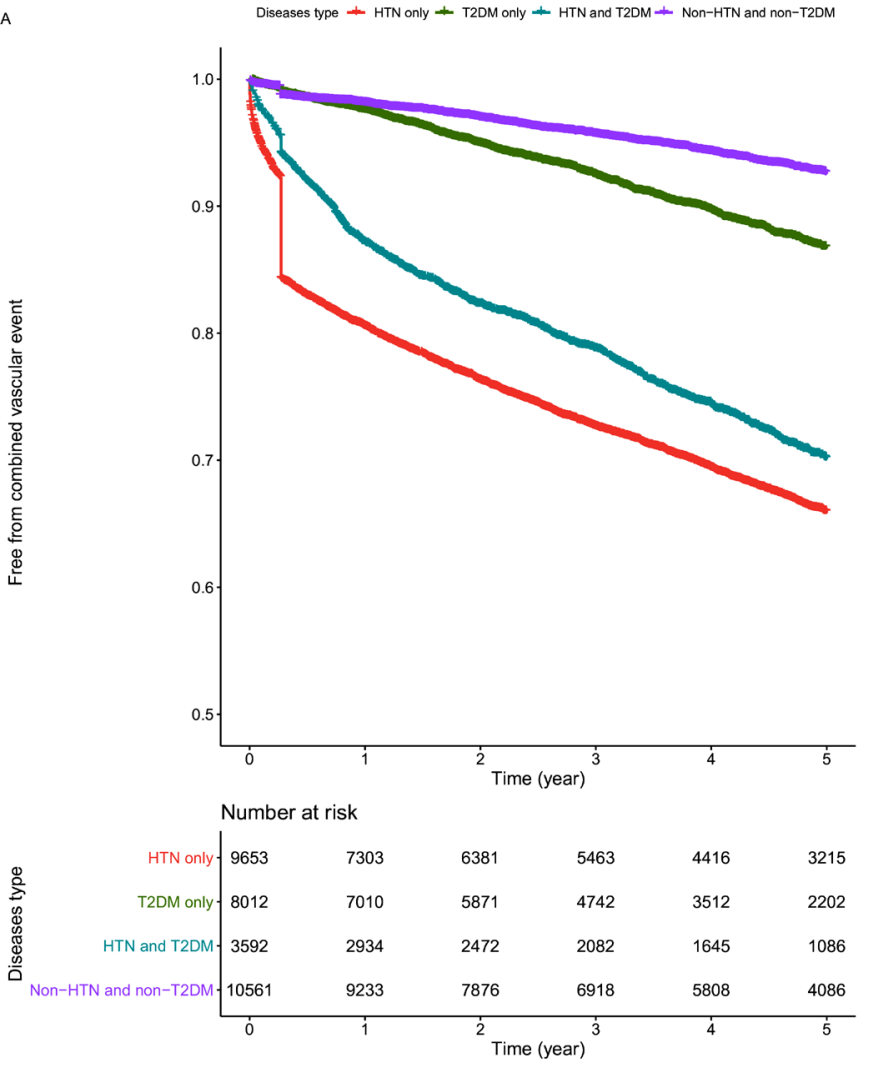

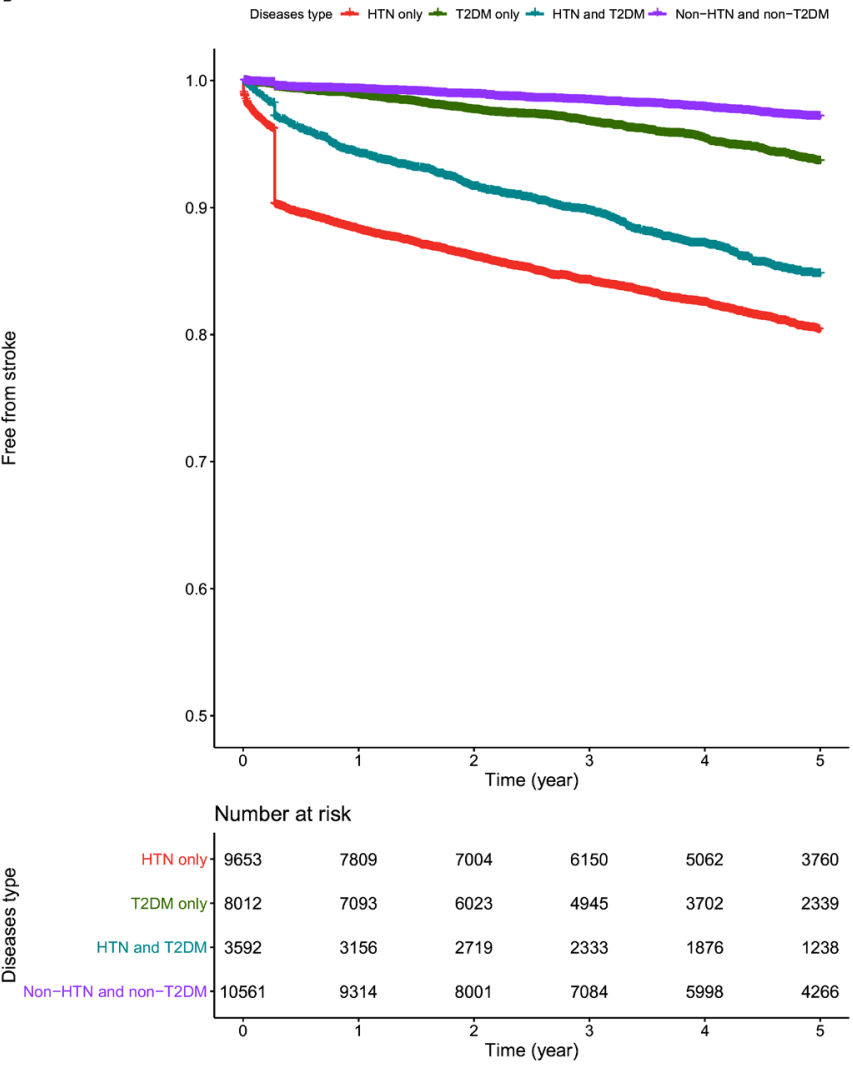

Figure 1 Kaplan-Meier survival curve of combined vascular events (A) and stroke (B) among different groups. HTN, hypertension; T2DM, type 2 diabetes mellitus.

the first diagnosis of T2DM and/or HTN. Patients were excluded as follows: missing sex information; had been diagnosed with stroke, myocardial infarction, coronary heart disease, heart failure; had received coronary artery bypass grafting or percutaneous coronary intervention before the first diagnosis of T2DM and/or HTN and had abnormal kidney function (normal range for serum creatinine: $54-106 \mu \mathrm{mol} / \mathrm{L}$ for men; $44-97 \mu \mathrm{mol} / \mathrm{L}$ for women). We also included non-T2DM and non-HTN patients who underwent the baseline examination but were not from the obstetrics and gynaecology department, cancer department, neurology department or cardiology department. Authorisation for the SuValue database was obtained when the database was set up, so neither ethics review nor written informed patient consent were needed for this analysis. In addition, all patients' EMRs were deidentified and anonymised when the SuValue database was constructed.

\section{Baseline parameters}

Sex, age, triglyceride, total cholesterol, high-density lipoprotein cholesterol (HDL-C), low-density lipoprotein cholesterol (LDL-C), serum creatinine, fasting blood glucose, serum insulin, proteinuria and medication (antidiabetics and antihypertensive medications) at baseline were captured from EMRs before or within 3 months of the first diagnosis of T2DM and/or HTN.

\section{OUTCOMES}

We examined two outcomes of interest: combined vascular events (VEs) and stroke. Combined VEs include stroke, myocardial infarction, coronary heart disease, heart failure and coronary bypass and percutaneous coronary intervention. The outcomes were defined as the first event or last record before 31 December 2019 among the event-free cases according to the diagnosis in the patients' EMRs.

\section{Statistical analyses}

Categorical variables were described using frequencies and percentages. Continuous variables were described using the mean (SD) if normally distributed or as the median IQR if not. Baseline characteristics were compared using analysis of variance or $\chi^{2}$ tests. KaplanMeier survival analysis of the incidence of combined VEs and stroke according to the presence of T2DM and/or HTN was performed. A Cox proportional hazards model was used to assess the association between diseases and each outcome, which was adjusted for cardiovascular risk factors, including sex, age, triglycerides, total cholesterol, HDL-C and LDL-C. Unadjusted, sex-adjusted and ageadjusted and cardiovascular risk factor-adjusted HRs were calculated. A $p<0.05$ (two-sided) was considered statistically significant. All statistical analyses were performed using SAS V.9.4 (SAS Institute, Cary, NC, USA). 
Table 2 Association of type 2 diabetes mellitus (T2DM) and/or hypertension (HTN) with combined vascular events and stroke compared with the non-T2DM and non-HTN

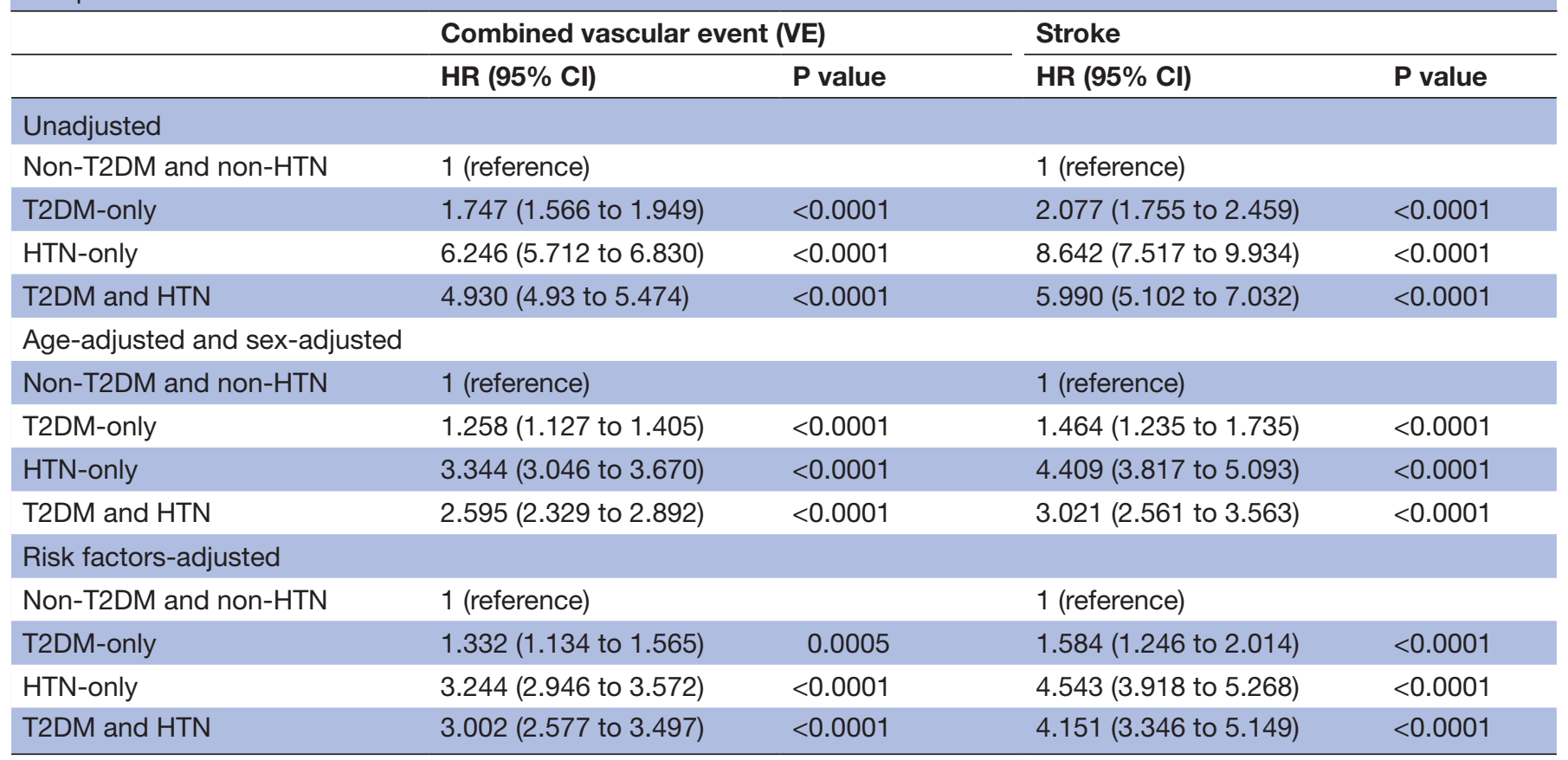

Risk factors include age, sex, triglycerides, total cholesterol, high-density lipoprotein cholesterol, low-density lipoprotein cholesterol. HTN, hypertension; T2DM, type 2 diabetes mellitus; VE, combined vascular event.

\section{Public and patient involvement}

Neither patients nor the public were involved in the design, conduct, reporting or dissemination plans of this research.

\section{RESULTS}

In the current study, 8012 patients with T2DM-only, 9653 patients with HTN-only and 3592 patients with both T2DM and HTN and 10561 patients without T2DM or HTN were selected from the SuValue database. The median follow-up duration was $4.1(2.4,4.9)$ years. The general characteristics of the study population at baseline are presented in table 1. Comparing baseline characteristics between the four groups revealed significant differences, except in total cholesterol $(p=0.3506)$ and serum insulin ( $\mathrm{p}=0.6502)$.

Kaplan-Meier analyses demonstrated increased combined VE and stroke risk in the HTN group (figure 1). In the unadjusted models, compared with non-HTN and non-T2DM, T2DM had an HR of 1.747 for combined VEs (95\% CI 1.566 to $1.949, \mathrm{p}<0.0001$ ) and 2.077 for stroke (95\% CI 1.755 to $2.459, \mathrm{p}<0.0001)$. Further adjustment with age and sex and then with cardiovascular risk factors attenuated the association of T2DM with combined VE and stroke risk (HR 1.332, 95\% CI 1.134 to 1.565 and HR 1.584, 95\% CI 1.246 to 2.014, respectively; $\mathrm{p}<0.001$, table 2).

Kaplan-Meier analyses demonstrated an increased combined VE and stroke risk in the HTN group (figure 1). The HTN-only group had a more than 6-fold increased risk of combined VE (95\% CI 5.712 to 6.830) and a more than eightfold increased risk of stroke (95\% CI 7.517 to 9.934) in unadjusted models (all Ps $<0.0001$ ). These associations were attenuated but still significant after adjustment for age and sex and then for other major cardiovascular risk factors for both combined VE and stroke risk (HR 3.244, 95\% CI 2.946 to 3.572 and HR $4.543,95 \%$ CI 3.918 to 5.268 , respectively; all Ps $<0.0001$, table 2 ).

Kaplan-Meier analyses demonstrated increased combined VE and stroke risk in the HTN and T2DM groups (figure 1). For combined VEs, the HR of the HTN and T2DM group was 4.93 (95\% CI 4.93 to 5.474). The HTN and T2DM groups had an increased risk of stroke to 5.990 (95\% CI 5.102 to 7.032$)$ in the unadjusted models (all Ps <0.0001). These associations were attenuated but still significant after adjustment for age and sex and then other major cardiovascular risk factors for both combined VE risk and stroke risk (HR 3.002, 95\% CI 2.577 to 3.497 and HR 4.151, 95\% CI 3.346 to 5.149, respectively; all Ps $<0.0001$, table 2).

Compared with the HTN-only group, the unadjusted HRs for combined VE risk were 0.789 and 0.693 for stroke risk in both the T2DM and HTN groups (all Ps $<0.0001$ ). However, after adjustment for major cardiovascular risk factors, there was no significantly reduced risk for either the T2DM or HTN group compared with the HTN-only group (table 3 ).

In the unadjusted model, the HTN and T2DM group and the HTN-only group had a higher combined VE and stroke risk than the T2DM-only group. After adjustment 
Table 3 Association of type 2 diabetes mellitus (T2DM) and hypertension (HTN) with combined vascular events and stroke compared with HTN-only

\begin{tabular}{|c|c|c|c|c|}
\hline & \multicolumn{2}{|c|}{ Combined vascular event (VE) } & \multicolumn{2}{|l|}{ Stroke } \\
\hline & HR (95\% Cl) & $P$ value & HR (95\% Cl) & $P$ value \\
\hline \multicolumn{5}{|l|}{ Unadjusted } \\
\hline HTN-only & 1 (reference) & & 1 (reference) & \\
\hline T2DM and HTN & 0.789 (0.732 to 0.851$)$ & $<0.0001$ & 0.693 (0.624 to 0.769$)$ & $<0.0001$ \\
\hline \multicolumn{5}{|c|}{ Age-adjusted and sex-adjusted } \\
\hline HTN-only & 1 (reference) & & 1 (reference) & \\
\hline T2DM and HTN & $0.776(0.720$ to 0.836$)$ & $<0.0001$ & 0.685 (0.617 to 0.761$)$ & $<0.0001$ \\
\hline \multicolumn{5}{|c|}{ Risk factors-adjusted } \\
\hline HTN-only & 1 (reference) & & 1 (reference) & \\
\hline T2DM and HTN & 0.925 (0.814 to 1.052$)$ & 0.2352 & 0.914 (0.771 to 1.082$)$ & 0.2959 \\
\hline
\end{tabular}

Risk factors include age, sex, triglycerides, total cholesterol, high-density lipoprotein cholesterol, low-density lipoprotein cholesterol. HTN, hypertension; T2DM, type 2 diabetes mellitus; VE, combined vascular event.

for age and sex and major cardiovascular risk factors, both the HTN and T2DM groups and the HTN-only group were still significantly associated with combined VE and stroke risk (table 4$)$.

\section{DISCUSSION}

In the present study, having HTN and/or T2DM was significantly associated with combined VE and stroke before and after adjustment for major cardiovascular risk factors compared with the non-T2DM and non-HTN. The association of T2DM with the risk of both combined $\mathrm{VE}$ and stroke attenuated after adjusting for major cardiovascular risk factors. In addition, the HTN-only group had a higher combined VE and stroke risk than the T2DM-only group.

To our knowledge, our study is the first to investigate the combined effect of HTN and T2DM in a large cohort in a real-world setting in Chinese patients. Several studies investigating the different impacts of HTN and diabetes on cardiovascular disease incidence and mortality have been conducted in Finland, America and Japan. ${ }^{21-23} 26$ HTN and/or T2DM were associated with an increased risk of combined VE and stroke in this study. The HR for cardiovascular disease was approximately two in

Table 4 Association of type 2 diabetes mellitus (T2DM) and/or hypertension (HTN) with combined vascular events and stroke compared with T2DM-only

\begin{tabular}{|c|c|c|c|c|}
\hline & \multicolumn{2}{|c|}{ Combined vascular event (VE) } & \multicolumn{2}{|l|}{ Stroke } \\
\hline & HR (95\% Cl) & $P$ value & HR (95\% Cl) & $P$ value \\
\hline \multicolumn{5}{|l|}{ Unadjusted } \\
\hline T2DM-only & 1 (reference) & & 1 (reference) & \\
\hline HTN-only & 3.575 (3.296 to 3.877$)$ & $<0.0001$ & 4.160 (3.701 to 4.675$)$ & $<0.0001$ \\
\hline T2DM and HTN & 2.821 (2.558 to 3.112 ) & $<0.0001$ & 2.883 (2.504 to 3.321$)$ & $<0.0001$ \\
\hline \multicolumn{5}{|l|}{$\begin{array}{l}\text { Age- } \\
\text { adjusted } \\
\text { and sex-adjusted }\end{array}$} \\
\hline T2DM-only & 1 (reference) & & 1 (reference) & \\
\hline HTN-only & 2.657 (2.447 to 2.885$)$ & $<0.0001$ & 3.011 (2.676 to 3.389$)$ & $<0.0001$ \\
\hline T2DM and HTN & 2.062 (1.868 to 2.276$)$ & $<0.0001$ & 2.063 (1.789 to 2.379$)$ & $<0.0001$ \\
\hline \multicolumn{5}{|c|}{ Risk factors-adjusted } \\
\hline T2DM-only & 1 (reference) & & 1 (reference) & \\
\hline HTN-only & 2.435 (2.113 to 2.805$)$ & $<0.0001$ & 2.868 (2.341 to 3.513$)$ & $<0.0001$ \\
\hline T2DM and HTN & 2.253 (1.876 to 2.706$)$ & $<0.0001$ & 2.620 (2.031 to 3.380$)$ & $<0.0001$ \\
\hline
\end{tabular}

Risk factors include age, sex, triglycerides, total cholesterol, high-density lipoprotein cholesterol and low-density lipoprotein cholesterol.

.HTN, hypertension; T2DM, type 2 diabetes mellitus; VE, combined vascular event. 
the T2DM-only group, which was lower than the three reported in the Framingham cohort of 1952-1974. ${ }^{11}$ Both HTN and T2DM have been shown to be strong risk factors for cardiovascular disease mortality. ${ }^{3}$

In unadjusted analyses and adjustment models for major cardiovascular risk factors, we observed that patients with both T2DM and HTN showed an increased risk of combined VE and stroke compared with those with T2DM alone. These results suggest that HTN confers an enhanced risk of cardiovascular disease. Similarly, the coexistence of HTN and T2DM conferred an increased risk of cardiovascular disease incidence compared with T2DM-only. ${ }^{3} 27$

After adjustment for major cardiovascular risk factors, patients with T2DM and HTN did not have a significantly increased combined $\mathrm{VE}$ and stroke risk compared with patients with HTN-only. In this study, we included patients with HTN and/or T2DM who had medication records. Antihypertension treatment, such as ACE inhibitors, calcium-channel blockers, or $\beta$ blockers, has been shown to reduce the risk of cardiovascular diseases according to several randomised trials and meta-analysis studies. ${ }^{28}{ }^{29} \mathrm{~A}$ meta-analysis demonstrated that antihypertensive treatment decreased the risk of cardiovascular disease and stroke among patients with a history of cardiovascular disease without HTN.$^{30}$ Thus, combined T2DM and HTN did not increase the risk of combined VE and stroke compared with HTN, which may be due to the antihypertensive treatment in this population.

In addition, we also observed that the $\mathrm{HTN}$-only group was more associated with combined VE and stroke risk than the T2DM group. The results were similar to a previous report that HTN alone was more related to allcause and atherosclerotic cardiovascular disease than T2DM alone in community-dwelling older adults. ${ }^{21} \mathrm{~A}$ study performed in older Iranian adults showed that T2DM alone increased the coronary heart disease incidence, stroke incidence and all-cause mortality compared with HTN alone. ${ }^{24}$ Thus, a prospective study is necessary for further analysis.

The current study has several significant strengths. First, this study included a large number of patients. Second, data about cardiovascular risk factors were collected in this study. However, there were several limitations in this study. First, Body Mass Index and lifestyle factors, such as smoking and alcohol consumption, were not recorded in the EMRs. Second, mortality data could not be accessed through the HIS system of hospitals.

\section{CONCLUSIONS}

In summary, HTN and/or T2DM was strongly associated with an increased risk of combined VE and stroke independent of conventional cardiovascular risk factors. T2DM does not seem to be a risk factor for combined VE and stroke in HTN patients after adjusting for major cardiovascular risk factors. HTN had a significantly higher combined VE and stroke risk than T2DM. However, a prospective study investigating the impact of HTN and/or T2DM on combined VE and stroke risk needs to be performed.

Contributors $\mathrm{HM}$ and $\mathrm{YL}$ designed the study, analysed the data and wrote the first draft of the manuscript. HM, YL, JL and YD revised it critically for important intellectual content and approved the final version. HM accepts full responsibility for the finished work and/or the conduct of the study, had access to the data, and controlled the decision to publish.

Funding The authors have not declared a specific grant for this research from any funding agency in the public, commercial or not-for-profit sectors.

Competing interests None declared.

Patient and public involvement Patients and/or the public were not involved in the design, or conduct, or reporting or dissemination plans of this research.

Patient consent for publication Not required.

Ethics approval All analyses were performed based on the SuValue database. Authorisation for the SuValue database was obtained when the database was set up, so neither ethics review or written informed patient consent were needed for this analysis. In addition, all patients' electronic medical records (EMRs) were deidentified and anonymised when the SuValue database was constructed.

Provenance and peer review Not commissioned; externally peer reviewed.

Data availability statement Data may be obtained from a third party and are not publicly available. All data relevant to the study are included in the article were obtained from SuValue database which belongs to a third party and is not publicly available.

Open access This is an open access article distributed in accordance with the Creative Commons Attribution 4.0 Unported (CC BY 4.0) license, which permits others to copy, redistribute, remix, transform and build upon this work for any purpose, provided the original work is properly cited, a link to the licence is given, and indication of whether changes were made. See: https://creativecommons.org/ licenses/by/4.0/.

ORCID iD

Hongshan Ma http://orcid.org/0000-0002-4029-4774

\section{REFERENCES}

1 Society CD. Guidelines for the prevention and treatment of type 2 diabetes in China (2017 edition). J Chin J Diabetes 2018;10:4-67.

2 Li Y, Teng D, Shi X, et al. Prevalence of diabetes recorded in mainland China using 2018 diagnostic criteria from the American diabetes association: national cross sectional study. BMJ 2020;369:m997.

3 Ferrannini E, Cushman WC. Diabetes and hypertension: the bad companions. Lancet 2012;380:601-10.

4 Sun D, Zhou T, Heianza Y, et al. Type 2 diabetes and hypertension. Circ Res 2019;124:930-7.

5 Rask-Madsen C, Li Q, Freund B, et al. Loss of insulin signaling in vascular endothelial cells accelerates atherosclerosis in apolipoprotein E null mice. Cell Metab 2010;11:379-89.

6 Rask-Madsen C, Buonomo E, Li Q, et al. Hyperinsulinemia does not change atherosclerosis development in apolipoprotein $\mathrm{E}$ null mice. Arterioscler Thromb Vasc Biol 2012;32:1124-31.

7 , Sarwar N, Gao P, et al, Emerging Risk Factors Collaboration. Diabetes mellitus, fasting blood glucose concentration, and risk of vascular disease: a collaborative meta-analysis of 102 prospective studies. Lancet 2010;375:2215-22.

8 Huxley R, Barzi F, Woodward M. Excess risk of fatal coronary heart disease associated with diabetes in men and women: meta-analysis of 37 prospective cohort studies. BMJ 2006;332:73-8.

9 Schramm TK, Gislason GH, Køber L, et al. Diabetes patients requiring glucose-lowering therapy and nondiabetics with a prior myocardial infarction carry the same cardiovascular risk: a population study of 3.3 million people. Circulation 2008;117:1945-54.

10 Almdal T, Scharling H, Jensen JS, et al. The independent effect of type 2 diabetes mellitus on ischemic heart disease, stroke, and death: a population-based study of 13,000 men and women with 20 years of follow-up. Arch Intern Med 2004;164:1422-6.

11 Fox CS. Cardiovascular disease risk factors, type 2 diabetes mellitus, and the framingham heart study. Trends Cardiovasc Med 2010;20:90-5.

12 Fox CS, Coady S, Sorlie PD, et al. Trends in cardiovascular complications of diabetes. JAMA 2004;292:2495-9. 
13 Hypertension in diabetes study (HDS): I. prevalence of hypertension in newly presenting type 2 diabetic patients and the association with risk factors for cardiovascular and diabetic complications. $J$ Hypertens 1993;11:309-17.

14 Lewington S, Clarke R, Qizilbash N, et al. Age-specific relevance of usual blood pressure to vascular mortality: a meta-analysis of individual data for one million adults in 61 prospective studies. Lancet 2002;360:1903-13.

15 Britton KA, Gaziano JM, Djoussé L. Normal systolic blood pressure and risk of heart failure in US male physicians. Eur $J$ Heart Fail 2009;11:1129-34.

16 Lawes CMM, Rodgers A, Bennett DA, et al. Blood pressure and cardiovascular disease in the Asia Pacific region. J Hypertens 2003;21:707-16.

17 Brown DW, Giles WH, Greenlund KJ. Blood pressure parameters and risk of fatal stroke, NHANES II mortality study. Am J Hypertens 2007;20:338-41.

18 Aronow WS, Fleg JL, Pepine CJ, et al. ACCF/AHA 2011 expert consensus document on hypertension in the elderly: a report of the American College of cardiology Foundation Task force on clinical expert consensus documents developed in collaboration with the American Academy of Neurology, American geriatrics Society, American Society for preventive cardiology, American Society of hypertension, American Society of nephrology, association of black cardiologists, and European Society of hypertension. J Am Soc Hypertens 2011;5:259-352.

19 Ikeda A, Iso H, Yamagishi K, et al. Blood pressure and the risk of stroke, cardiovascular disease, and all-cause mortality among Japanese: the JPHC study. Am J Hypertens 2009;22:273-80.

20 Lotfaliany M, Akbarpour S, Mozafary A, et al. Hypertension phenotypes and incident cardiovascular disease and mortality events in a decade follow-up of a middle East cohort. J Hypertens 2015;33:1153-61.

21 Oh J-Y, Allison MA, Barrett-Connor E. Different impacts of hypertension and diabetes mellitus on all-cause and cardiovascular mortality in community-dwelling older adults: the Rancho bernardo study. J Hypertens 2017;35:55-62.

22 Hu G, Jousilahti P, Tuomilehto J. Joint effects of history of hypertension at baseline and type 2 diabetes at baseline and during follow-up on the risk of coronary heart disease. Eur Heart $J$ 2007;28:3059-66.

23 Kokubo Y, Okamura T, Watanabe M, et al. The combined impact of blood pressure category and glucose abnormality on the incidence of cardiovascular diseases in a Japanese urban cohort: the Suita study. Hypertens Res 2010;33:1238-43.

24 Zafari N, Asgari S, Lotfaliany M, et al. Impact of hypertension versus diabetes on cardiovascular and all-cause mortality in Iranian older adults: results of 14 years of follow-up. Sci Rep 2017;7:14220.

25 Wang C, Gao Y, Zhu L, et al. Treatment patterns in patients with newly diagnosed type 2 diabetes in China: a retrospective, longitudinal database study. Clin Ther 2019;41:1440-52.

26 Hu G, Sarti C, Jousilahti P, et al. The impact of history of hypertension and type 2 diabetes at baseline on the incidence of stroke and stroke mortality. Stroke 2005;36:2538-43.

27 Chen G, McAlister FA, Walker RL, et al. Cardiovascular outcomes in Framingham participants with diabetes: the importance of blood pressure. Hypertension 2011;57:891-7.

28 Patel A, MacMahon S, et al, ADVANCE Collaborative Group. Effects of a fixed combination of perindopril and indapamide on macrovascular and microvascular outcomes in patients with type 2 diabetes mellitus (the advance trial): a randomised controlled trial. Lancet 2007;370:829-40.

29 Turnbull F, Neal B, Algert C, et al. Effects of different blood pressurelowering regimens on major cardiovascular events in individuals with and without diabetes mellitus: results of prospectively designed overviews of randomized trials. Arch Intern Med 2005;165:1410-9.

30 Thompson AM, Hu T, Eshelbrenner CL, et al. Antihypertensive treatment and secondary prevention of cardiovascular disease events among persons without hypertension: a meta-analysis. JAMA 2011;305:913-22. 